\title{
ENTIRE FUNCTIONS THAT SHARE TWO VALUES
}

\author{
PING LI
}

\begin{abstract}
In this paper, we find all the possible forms of two nonconstant entire functions $f$ and $g$ that share two values counting multiplicities. As applications, we generalize some known results and confirm a conjecture proposed by Osgood-Yang.
\end{abstract}

\section{Introduction and results}

Let $f$ be a meromorphic functions defined on the complex plane C. In this paper, we shall use the standard notations in Nevanlinna's value distribution theory of meromorphic functions such as the characteristic function $T(r, f)$, the counting function of the poles $N(r, f)$, and the proximity function $m(r, f)$ (see, e.g., [3]). As usual, $\bar{N}(r, f)$ is the reduced counting function of the poles of $f$, i.e., the counting function which count every poles only once ignoring the multiplicities. We denote by $N_{k)}(r, f)$ the counting function of the poles of $f$ of multiplicities $\leq k$, and denote by $N_{(k}(r, f)$ the counting function of the poles of $f$ of multiplicities $\geq k$. The notation $S(r, f)$ is defined to be any quantity satisfying $S(r, f)=o(T(r, f))$ as $r \rightarrow \infty$ possibly outside a set of $r$ of finite linear measure. Let $f$ and $g$ be two nonconstant meromorphic functions, and $a$ be a value in $\mathbf{C}$. We say that $f$ and $g$ share $a$ IM $(\mathrm{CM})$ provided that $f(z)-a$ and $g(z)-a$ have same zeros ignoring multiplicities (counting multiplicities). It is well known (see [2]) that the Nevanlinna characteristic functions $T(r, f)$ and $T(r, g)$ satisfy the following relation:

$$
T(r, f)=T(r, g)+S(r, g)
$$

provided that $f$ and $g$ share four values IM. In 1976, Osgood-Yang [8] proved that if $f$ and $g$ are two nonconstant entire functions of finite order, and share two distinct finite values $\mathrm{CM}$, then $T(r, f) \sim T(r, g)(r \rightarrow \infty)$. Osgood-Yang conjectured that the restriction for the order in this result can be removed. There have been published many other results related to entire functions sharing two values $\mathrm{CM}$, or meromorphic functions sharing three values $\mathrm{CM}$ (see $[4,5,6]$ and $[9,10,11])$.

AMS(2000) Subject Classification. 30D35.

Keywords and phrases. meromorphic function, entire function, value sharing.

Received January 11, 2005; revised April 22, 2005. 
In 1998, the author proved the following result.

THEOREM A ([5]). Let $f$ and $g$ be nonconstant meromorphic functions sharing $0,1, \infty C M$. Suppose additionally that $f$ is not a Möbius transformation of $g$ and that there exists an $a \neq 0,1, \infty$ and a positive constant $c$ such that

$$
T(r, f) \leq c \bar{N}_{(2}\left(r, \frac{1}{f-a}\right)+S(r, f),
$$

then there exist a nonconstant entire function $\gamma$, a nonzero constant $\lambda$ and two integers $s, t(t>0)$ which are mutually prime, such that

$$
\begin{gathered}
f=\frac{e^{t \gamma}-1}{\lambda e^{-s \gamma}-1}, \quad g=\frac{e^{-t \gamma}-1}{\frac{1}{\lambda} e^{s \gamma}-1}, \\
\frac{(1-a)^{s+t}}{a^{t}}=\lambda^{t} \frac{(1-\theta)^{s+t}}{\theta^{t}},
\end{gathered}
$$

with $\theta=-\frac{t}{s} \neq 1, a$.

The author also gave all the possible meromorphic functions $f$ and $g$ if the inequality in (1) is replaced by

$$
\bar{N}_{1)}\left(r, \frac{1}{f-a}\right)=S(r, f) .
$$

In 2003, W.-R. Lü and H.-X. Yi considered another conditions different from that in (1) and (2), and proved the following results.

THEOREM B ([6]). Let $f$ and $g$ be two distinct meromorphic functions sharing 0,1 and $\infty$ CM. Suppose that

$$
\limsup _{r \rightarrow \infty} \frac{N_{1)}(r, f)+N_{1)}\left(r, \frac{1}{f}\right)}{T(r, f)}<1 .
$$

Then

$$
f=\frac{e^{s \gamma}-1}{e^{-(k+1-s) \gamma}-1}, \quad g=\frac{e^{-s \gamma}-1}{e^{(k+1-s) \gamma}-1},
$$

where $s$ and $k$ are positive integers $(1 \leq s \leq k)$ such that $(s, k+1)=1$, and $\gamma$ is a nonconstant entire function.

THEOREM C ([6]). Let $f$ and $g$ be two meromorphic functions sharing 0,1 and $\infty C M$, and suppose that $N_{1)}(r, f)=S(r, f)$. If 


$$
\limsup _{r \rightarrow \infty} \frac{N_{1)}\left(r, \frac{1}{f-1}\right)+N_{1)}\left(r, \frac{1}{f}\right)}{T(r, f)}<2, \quad(r \in I),
$$

then $f$ and $g$ assume one of the following forms:

(i) $f=e^{k \gamma}+e^{(k-1) \gamma}+\cdots+e^{\gamma}+1, g=e^{-k \gamma}+e^{-(k-1) \gamma}+\cdots+e^{-\gamma}+1$;

(ii) $f=-e^{k \gamma}-e^{(k-1) \gamma}-\cdots-e^{\gamma}, g=-e^{-k \gamma}-e^{-(k-1) \gamma}-\cdots-e^{-\gamma}$,

where $k$ is a positive integer and $\gamma$ is a nonconstant entire function.

In this paper, we shall find all the possible forms of two nonconstant entire functions $f$ and $g$ that share two finite values CM. In fact, we shall prove the following result.

THEOREM 1. Suppose that $f$ and $g$ are two distinct nonconstant entire functions. If $f$ and $g$ share the values 0 and $1 C M$, then they assume one of the following cases:

(i) $f=c\left(1-e^{\zeta}\right), g=(1-c)\left(1-e^{-\zeta}\right)$;

(ii) $f=e^{-n \zeta} \sum_{j=0}^{n} e^{j \zeta}, g=\sum_{j=0}^{n} e^{j \zeta}, n=1,2, \ldots$;

(iii) $f=-e^{-(n+1) \zeta} \sum_{j=0}^{n} e^{j \zeta}, g=-e^{\zeta} \sum_{j=0}^{n} e^{j \zeta}, n=0,1,2, \ldots$,

where $c(\neq 0,1)$ is a constant, and $\zeta$ is a nonconstant entire function.

Theorem 1 can be generalized to the case (see, Section 3) that $f$ and $g$ are meromorphic functions with the conditions $\bar{N}(r, f)=S(r, f)$ and $\bar{N}(r, g)=$ $S(r, g)$. By Theorem 1, we can get the following results easily. The first one gives a positive answer to Osgood-Yang's conjecture, and the others extend some known results which had some restrictions for the order.

COROLlaRY 1. If nonconstant entire functions $f$ and $g$ share two values counting multiplicities, then $T(r, f)=T(r, g)+O(1)$.

COROLlary 2. Suppose that $f$ and $g$ are two distinct nonconstant entire functions sharing the values 0 and $1 C M$. If $\delta(0, f)>0$, and if $\delta(0, f) \neq 1 / p$ for any integer $p(\geq 2)$, then $f=e^{\zeta}$ and $g=e^{-\zeta}$, where $\zeta$ is a nonconstant entire function.

COROLlaRY 3. Suppose that $f$ and $\alpha$ are nonconstant entire functions, and $a_{1}, a_{2}$ are two nonzero constant. If $f$ share 0 and $1 C M$ with $g=a_{1} e^{\alpha}+a_{2} e^{-\alpha}$, then $f=g$.

\section{Lemmas and proof of the main result}

The following two lemmas will be used in the proof of the main theorem.

LEMMA 1 ([4]). Let $f_{1}$ and $f_{2}$ be two nonconstant meromorphic functions satisfying 


$$
\bar{N}\left(r, f_{i}\right)+\bar{N}\left(r, \frac{1}{f_{i}}\right)=S(r), \quad i=1,2 .
$$

If $f_{1}^{s} f_{2}^{t}-1$ is not identically zero for all integers $s$ and $t(|s|+|t|>0)$, then for any positive number $\varepsilon$, we have

$$
N_{0}\left(r, 1 ; f_{1}, f_{2}\right) \leq \varepsilon T(r)+S(r),
$$

where $N_{0}\left(r, 1 ; f_{1}, f_{2}\right)$ denotes the reduced counting function of $f_{1}$ and $f_{2}$ related to the common 1-points, which counts such points only once ignoring multiplicities, and $T(r)=T\left(r, f_{1}\right)+T\left(r, f_{2}\right), S(r)=o(T(r))$ as $r \rightarrow \infty$, except for a set of $r$ of finite linear measure.

LEMMA 2 ([1] or [7]). If $f$ and $g$ are nonconstant meromorphic functions sharing 0, 1 and $\infty C M$ and $f$ is not a Möbius transformation of $g$, then

$$
T(r, f)+T(r, g)=N(r, f)+N\left(r, \frac{1}{f}\right)+N\left(r, \frac{1}{f-1}\right)+N_{0}(r)+S(r),
$$

where $N_{0}(r)$ denotes the reduced counting function of the zeros of $f-g$ which are not the 0-points, 1-points or poles of $f$ and $g$, and $S(r):=S(r, f)=S(r, g)$.

For convenience, we introduce the notation $S^{*}(r, f)$ which is defined to be any quantity such that for any positive number $\varepsilon$ there exists a $S(r, f)$ satisfying the following inequality:

$$
\left|S^{*}(r, f)\right| \leq \varepsilon T(r, f)+S(r, f) .
$$

Suppose that $\mathscr{M}(\mathbf{C})$ is the set of all meromorphic functions on C. For $f \in \mathscr{M}(\mathbf{C})$, Let

$$
\begin{aligned}
S(f) & =\{g \in \mathscr{M}(\mathbf{C}): T(r, g)=S(r, f)\}, \\
S^{*}(f) & =\left\{g \in \mathscr{M}(\mathbf{C}): T(r, g)=S^{*}(r, f)\right\} .
\end{aligned}
$$

It is obvious that both $S(f)$ and $S^{*}(f)$ are fields of functions, which are closed under products and differentiating, and $S(f) \subset S^{*}(f)$. It is easily seen that we can not find any set $I$ of infinite linear measure such that $T(r, f) \leq S^{*}(r, f)$, $r \in I$.

Now we prove the main result. Suppose that $f$ and $g$ are two distinct nonconstant entire functions, and share $0,1 \mathrm{CM}$. Then there exists two entire functions $\alpha$ and $\beta$ such that

$$
\frac{f}{g}=e^{\alpha}, \quad \frac{f-1}{g-1}=e^{\beta} .
$$

Since $f$ and $g$ share 0 and $1 \mathrm{CM}$, by Nevanlinna's second fundamental theorem, we get

$$
T(r, f) \leq 2 T(r, g)+S(r, f) \quad \text { and } \quad T(r, g) \leq 2 T(r, f)+S(r, g) .
$$


Therefore, an $S(r, f)$ is also an $S(r, g)$, and vice versa. If $e^{\alpha}=c$ is a constant, then it is easily seen that $c \neq 0,1$. Note that $f$ and $g$ share $1 \mathrm{CM}$. We see that both 1 and $c$ are the exceptional values of $f$. This is impossible. Hence $e^{\alpha}$ is not a constant. Similarly, $e^{\beta}$ is not a constant either.

If $e^{\alpha-\beta}=c$ is a constant, then $c \neq 1$, otherwise $f=g$. It follows from (3) that

$$
f=\frac{c}{c-1}\left(1-e^{\beta}\right), \quad g=\frac{1}{c-1}\left(e^{-\beta}-1\right) .
$$

Therefore, $f$ and $g$ assume the first form in Theorem 1 .

In the sequel, we suppose that $e^{\alpha}, e^{\beta}$ and $e^{\alpha-\beta}$ are not constants, and distinguish two cases below.

CASE 1. $\left(e^{-\beta}\right)^{s}-\left(e^{\alpha-\beta}\right)^{t}$ is not identically zero for any integers $s$ and $t$ $(|s|+|t|>0)$.

In this case, by Lemma 1 we see that

$$
\bar{N}_{0}\left(r, 1 ; e^{-\beta}, e^{\alpha-\beta}\right) \leq \varepsilon\left(T\left(r, e^{-\beta}\right)+T\left(r, e^{\alpha-\beta}\right)\right)+S(r, f)
$$

holds for any positive number $\varepsilon$. It follows from (3) that

$$
f=\frac{e^{\alpha-\beta}-e^{\alpha}}{e^{\alpha-\beta}-1}, \quad g=\frac{e^{-\beta}-1}{e^{\alpha-\beta}-1} .
$$

Since $f$ and $g$ are entire, we see that any 1-points of $e^{\alpha-\beta}$ is a common 1-points of $e^{-\beta}$ and $e^{\alpha-\beta}$. Therefore,

$$
\bar{N}\left(r, \frac{1}{e^{\alpha-\beta}-1}\right) \leq \bar{N}_{0}\left(r, 1 ; e^{-\beta}, e^{\alpha-\beta}\right) .
$$

This and (4) imply $\bar{N}\left(r, \frac{1}{e^{\alpha-\beta}-1}\right)=S^{*}(r, f)$. And thus

$$
T\left(r, e^{\alpha-\beta}\right)=S^{*}(r, f) .
$$

Since $N_{0}(r)$ is the reduced counting function which counts the zeros of $f-g$, but does not count 0 -points, 1-points or poles of $g$, we have

$$
N_{0}(r) \leq \bar{N}_{0}\left(r, 1 ; e^{-\beta}, e^{\alpha-\beta}\right) \leq S^{*}(r, f) .
$$

By Lemma 2, we get

$$
\begin{aligned}
T(r, f)+T(r, g) & =N\left(r, \frac{1}{f}\right)+N\left(r, \frac{1}{f-1}\right)+N(r, f)+N_{0}(r)+S(r, f) \\
& =N\left(r, \frac{1}{f}\right)+N\left(r, \frac{1}{g-1}\right)+S^{*}(r, f),
\end{aligned}
$$

which implies 


$$
m\left(r, \frac{1}{f}\right)+m\left(r, \frac{1}{g-1}\right)=S^{*}(r, f) .
$$

Let

$$
\varphi=\frac{f-g}{f(g-1)} .
$$

Then we have $N(r, \varphi)=0$. By (7), we have $m(r, \varphi)=S^{*}(r, f)$. Therefore,

$$
T(r, \varphi)=S^{*}(r, f) .
$$

It is obvious that $\varphi \neq 0,-1$ and $g=(1+\varphi) f /(1+\varphi f)$. Therefore, $N(r, 1 /$ $(\varphi f+1))=S^{*}(r, f)$. Let

$$
\psi=\frac{\varphi^{\prime} f+\varphi f^{\prime}}{\varphi f+1} .
$$

By the lemma of logarithmic derivative, we have $m(r, \psi)=S^{*}(r, f)$. Thus, $T(r, \psi)=S^{*}(r, f)$. Let

$$
\gamma=\frac{1}{e^{\alpha-\beta}-1}
$$

It follows from (6) that $T(r, \gamma)=S^{*}(r, f)$. By (5) and (11), we deduce that

$$
f=\gamma+1-\gamma e^{\alpha} \text {. }
$$

Therefore,

$$
\begin{aligned}
f^{\prime} & =\gamma^{\prime}-\left(\gamma^{\prime}+\gamma \alpha^{\prime}\right) e^{\alpha} \\
& =\gamma^{\prime}-\left(\gamma^{\prime}+\gamma \alpha^{\prime}\right)\left(\frac{\gamma+1-f}{\gamma}\right) \\
& =\gamma^{\prime}-\left(\frac{\gamma^{\prime}}{\gamma}+\alpha^{\prime}\right)(\gamma+1-f) .
\end{aligned}
$$

This and (10) imply

$$
\psi \varphi f+\psi=\left(\varphi^{\prime}+\varphi\left(\frac{\gamma^{\prime}}{\gamma}+\alpha^{\prime}\right)\right) f-\varphi\left(\frac{\gamma^{\prime}}{\gamma}+\alpha^{\prime} \gamma+\alpha^{\prime}\right) .
$$

If $\psi \varphi \neq \varphi^{\prime}+\varphi\left(\frac{\gamma^{\prime}}{\gamma}+\alpha^{\prime}\right)$, then the above equation implies $T(r, f)=S^{*}(r, f)$, which is impossible. Suppose $\psi \varphi=\varphi^{\prime}+\varphi\left(\frac{\gamma^{\prime}}{\gamma}+\alpha^{\prime}\right)$. We get

$$
\psi=\frac{\varphi^{\prime}}{\varphi}+\frac{\gamma^{\prime}}{\gamma}+\alpha^{\prime}
$$

Combining this and (10), we have 


$$
\frac{\varphi^{\prime} f+\varphi f^{\prime}}{\varphi f+1}=\frac{\varphi^{\prime}}{\varphi}+\frac{\gamma^{\prime}}{\gamma}+\alpha^{\prime}
$$

By integration, we get

$$
\varphi f+1=c(\varphi \gamma \alpha)
$$

where $c$ is a nonzero constant. This also implies $T(r, f)=S^{*}(r, f)$, a contradiction. Case 1 has been ruled out.

CASE 2. There exist two nonzero integers $s$ and $t$ such that $\left(e^{-\beta}\right)^{s}=\left(e^{\alpha-\beta}\right)^{t}$.

Without loss of generality, we assume $s>0$. Let $d$ be the maximal common factor of $s$ and $t$. Thus there exist two integers $u$ and $v$ such that $u s+v t=d$. Let $h=\left(e^{-\beta}\right)^{v / d}\left(e^{\alpha-\beta}\right)^{u / d}$. Then we have $e^{\alpha-\beta}=h^{s}$ and $e^{-\beta}=h^{t}$. If $s>d$, then any $e^{2 \pi i / s}$ point of $h$ is an 1-point of $e^{\alpha-\beta}$, but not an 1-point of $e^{-\beta}$. Note that any 1-point of $e^{\alpha-\beta}$ must be an 1-point of $e^{-\beta}$. Hence $s=d$. Therefore, $e^{-\beta}=\left(e^{\alpha-\beta}\right)^{n}$, where $n=t / d$. If $n>0$, then it follows from (5) that

$$
f=e^{-(n-1)(\alpha-\beta)} \sum_{j=0}^{n-1} e^{(\alpha-\beta) j}, \quad g=\sum_{j=0}^{n-1} e^{(\alpha-\beta) j} .
$$

Hence $f$ and $g$ assume the second case in Theorem 1. If $n<0$, then from (5) we get

$$
f=-e^{n(\beta-\alpha)} \sum_{j=0}^{-n-1} e^{(\beta-\alpha) j}, \quad g=-e^{\beta-\alpha} \sum_{j=0}^{-n-1} e^{(\beta-\alpha) j} .
$$

Hence $f$ and $g$ assume the third case in Theorem 1. This completes the proof of Theorem 1 .

\section{Concluding remark}

A meromorphic function $a(\not \equiv \infty)$ is called a small function with respect to $f$ provided that $T(r, a)=S(r, f)$. Let $f$ and $g$ be two nonconstant meromorphic functions, and let $a$ be a small function with respect to $f$ and $g$. Denote by $\bar{N}_{E}(r, f=a=g)$ the reduced counting function of the common $a$ points of $f$ and $g$ with the same multiplicities. We say that $f$ and $g$ share $a$ in the sense of $C M^{*}$, if

$$
\bar{N}\left(r, \frac{1}{f-a}\right)-\bar{N}_{E}(r, f=a=g)=S(r, f),
$$

and

$$
\bar{N}\left(r, \frac{1}{g-a}\right)-\bar{N}_{E}(r, f=a=g)=S(r, g) .
$$


By the arguments similar to that in the proof of Theorem 1, we can proof the following result.

THEOREM 2. Suppose that $f$ and $g$ are two distinct nonconstant meromorphic functions satisfying $\bar{N}(r, f)=S(r, f)$ and $\bar{N}(r, g)=S(r, g)$. If $f$ and $g$ share the values 0 and $1 C M^{*}$, then they assume one of the following cases:

(i) $f=c(1-h), g=(1-c)(1-1 / h)$;

(ii) $f=h^{-n} \sum_{j=0}^{n} h^{j}, g=\sum_{j=0}^{n} h^{j}, n=1,2, \ldots$;

(iii) $f=-h^{-(n+1)} \sum_{j=0}^{n} h^{j}, g=-h \sum_{j=0}^{n} h^{j}, n=0,1,2, \ldots$,

where $h$ is a nonconstant meromorphic function satisfying

$$
\bar{N}(r, h)+\bar{N}\left(r, \frac{1}{h}\right)=S(r, h),
$$

and $c(\not \equiv 0,1)$ is a small function of $h$.

\section{REFERENCES}

[1] G. Brosch, Eindeutigkeitssätze für meromorphe Funktionen, Thesis, Technical University of Aachen, 1989.

[ 2 ] G. G. Gundersen, Meromorphic functions that share three or four values, J. London Math. Soc. (2) 20 (1979), 457-466.

[ 3 ] W. K. Hayman, Meromorphic Functions, Clarendon Press, Oxford, 1964.

[4] P. Li AND C. C. YANG, On the characteristic of meromorphic functions that share three values CM, J. of Math. Anal. and Appl. 220 (1998), 132-145.

[ 5 ] P. LI, Meromorphic functions sharing three values or sets CM, Kodai Math. J. 21 (1998), $138-152$.

[6] W.-R. LÜ AND H.-X. YI, Unicity theorems of meromorphic functions that share three values, Ann. Polonici Math. 81 (2003), 131-138.

[7] E. Mues, Shared value problems for meromorphic functions, Value distribution theory and complex differential equations, Joensuu, 1994, Univ. Joensuu Publications in Sciences 35, $1995,17-43$.

[8] C. F. Osgood And C. C. Yang, On the quotient of two integral functions, J. Math. Anal. Appl. 54 (1976), 408-418.

[9] M. Ozawa, Unicity theorems for entire functions, J. d'Anal. Math. 30 (1976), 411-420.

[10] H. UedA, Unicity theorems for entire functions, Kodai Math. J. 3 (1980), 212-223.

[11] H. UEDA, Unicity theorems for meromorphic or entire functions, II, Kodai Math. J. 6 (1983), 26-36.

Department of Mathematics

University of ScI. \& TeCh. OF China

HeFei, ANHUi 230026

P. R. CHINA

E-mail: pli@ustc.edu.cn 